\title{
Development of An Android Application for Viewing Covid-19 Containment Zones and Monitoring Violators Who are Trespassing into It Using Firebase and Geofencing
}

\author{
Ranajoy Mallik $^{1}$ (D) Amlan Protim Hazarika ${ }^{1} \cdot$ Sudarshana Ghosh Dastidar ${ }^{1} \cdot$ Dilip Sing $^{1} \cdot$ Rajib Bandyopadhyay $^{1}$
}

Received: 29 April 2020 / Revised: 13 June 2020 / Accepted: 18 June 2020 / Published online: 1 July 2020

(C) Indian National Academy of Engineering 2020

\begin{abstract}
The World Health Organization has declared the outbreak of the novel coronavirus, Covid-19 as pandemic across the world. With its alarming surge of affected cases throughout the world, lockdown, and awareness (social distancing, use of masks etc.) among people are found to be the only means for restricting the community transmission. In a densely populated country like India, it is very difficult to prevent the community transmission even during lockdown without social awareness and precautionary measures taken by the people. Recently, several containment zones had been identified throughout the country and divided into red, orange and green zones, respectively. The red zones indicate the infection hotspots, orange zones denote some infection and green zones indicate an area with no infection. This paper mainly focuses on development of an Android application which can inform people of the Covid-19 containment zones and prevent trespassing into these zones. This Android application updates the locations of the areas in a Google map which are identified to be the containment zones. The application also notifies the users if they have entered a containment zone and uploads the user's IMEI number to the online database. To achieve all these functionalities, many tools, and APIs from Google like Firebase and Geofencing API are used in this application. Therefore, this application can be used as a tool for creating further social awareness about the arising need of precautionary measures to be taken by the people of India.
\end{abstract}

Keywords Covid-19 $\cdot$ Android $\cdot$ Geo-fencing $\cdot$ Firebase $\cdot$ Location tracking $\cdot$ Notification $\cdot$ IMEI number

\section{Introduction}

Currently there are several research works undergoing in the country to prevent Covid-19 cases from rising. Previously our country was importing medical kits like PPE (Personal Protection Kits), mask from outside, but now it has been successful in developing these kits. Along with taking initiatives to fight this disease, our country has also taken steps to make people aware of the disease. The news and media have a great part in creating this awareness by informing the public about the preventive measures that can keep them away from infection. Awareness among the people to carry out all the preventive measures can immensely help to reduce

Ranajoy Mallik

wranajoy@gmail.com

1 Department of Instrumentation and Electronics Engineering, Jadavpur University, Salt Lake Campus, Kolkata 700 098, India spread of the virus. The country has created containment zones throughout the cities wherever Covid-19 cases have been reported to prevent further spread of the virus. These containment zones have been kept isolated from the outside public to ensure no contamination occurs outside.

After more than 2 months of the lockdown, the government has relaxed some of the lockdown rules and has permitted reopening of government offices, bus and other road transportation facilities and shopping markets. People can move inside the city for work and other purposes. But the containment zones are still being kept isolated, and new containment zones are being formed wherever Covid-19 cases have been reported. These zones are highly contagious as droplets with virus coughed out from an unscreened asymptomatic patient can travel up to $8 \mathrm{~m}$ (Bahl et al. 2020). Though these containment zones are guarded by policemen, still there remains a chance that people might unknowingly step into them. In this situation where people can move in the city, these containment zones pose a risk of infection to these city dwellers. Therefore, informing people about the 
location of the containment zones can help them bypass and avoid these zones and thereby reduce the chance of community transmission.

In this paper, we focus on developing a mobile based application to provide information regarding the Covid-19 containment zones in West Bengal. The application further tracks the user's location and provides notification alert if the user has entered a containment zone. The application also provides daily Covid-19 case statistics to the users to keep them updated. The application is developed on Android SDK and uses Firebase Cloud Firestore to store the location data. Android's geofencing client is used to create geofences around the containment zones and notification manager is used to provide notifications. The application also uses RESTful web services to show the Covid-19 cases in West Bengal.

We have tested our application with different users in different locations across West Bengal and it works efficiently and is able to attain our target.

\section{Existing Apps in Google Playstore Related to Covid-19}

We have conducted a brief survey on the existing apps published in Google playstore which are related to Covid-19. Efforts have been made to include most of the apps in the survey. The summary of the survey is given in Table 1 which includes the name of the apps, the description of the apps given in Google Playstore by their developers and our comments on the apps after using them.

The survey shows that there are several apps developed in the country to fight and contain COVID-19. Most of the states of our country have their own apps with specific features and functionality to help their citizens to stop COVID19 spread, get medical assistance during a crisis, create awareness, and understand safety precautions. The study also shows that there are a limited number of apps which show the COVID-19 containment zones in the country or state and out of these none has the functionality of notifying and alerting the user when they have entered a containment zone. Therefore, no app in the Google Playstore is comparable with our proposed application because the idea behind the development of the proposed app is different. This highlights the novelty of the proposed app.

\section{Proposed Work}

The Android application shows the location of the containment zones to the users. It also notifies the user when he or she trespasses the boundary of a containment zone or stays in the containment zones (Fig. 1).

\section{Application Design}

There are mainly three activities in the application. The first activity consists of a welcome screen which is designed with images and information. Next activity is a screen displaying the instructions to operate the application and a disclaimer (Figs. 2,3). The third activity is a maps activity which shows all the containment zones in a google map (Fig. 4). This activity also has a bottom sheet which can be pulled up to show the real time Covid-19 statistic of West Bengal.

\section{Firebase Cloud Firestore}

The application uses Firestore which is a flexible and scalable database for mobile, web and server developments from Firebase and Google cloud platform (Cloud Firestore 2020). In Cloud Firestore, the mobile application supports serverless app architecture where the application connects to the Cloud Firestore database directly without any intermediate servers in between (Cloud Firestore SDKs and client libraries 2020). The application receives data from the database using WebSocket. The Web Socket transfers data at a higher speed than HTTP. The database transfers new data to the user as soon as it is updated. When changes are saved in the database then all connected clients receive the updated data almost instantly. The Cloud Firestore does not always fetch data from the database unless the data has been changed, it gathers previous data from the cache memory which also enables its offline functioning.

The Cloud Firestore features a NoSQL, document-oriented database (Cloud Firestore Data model 2020) and the data is stored in a JavaScript Object Notation (JSON) format. The location data are stored in documents, which are organized into collections. All the containment zones are stored in a collection in which each containment zone is represented as an individual document. Each document has four fields namely "Lat", "Long", "locationName" and "radius" for storing latitudes, longitudes, location names and radius respectively. Figure 5 shows the documentoriented Cloud Firestore database with data of few containment zones. The "radius" field in each document is used to indicate the radius of the containment zone. In the development stage, the billing plan for the database used here is the firebase spark plan which is the free plan provided by Firebase. This plan has few limitations like 50,000 and 20,000 reads and writes per day. These usage limits can be resolved by choosing a different paid plan from Firebase according to requirement. 


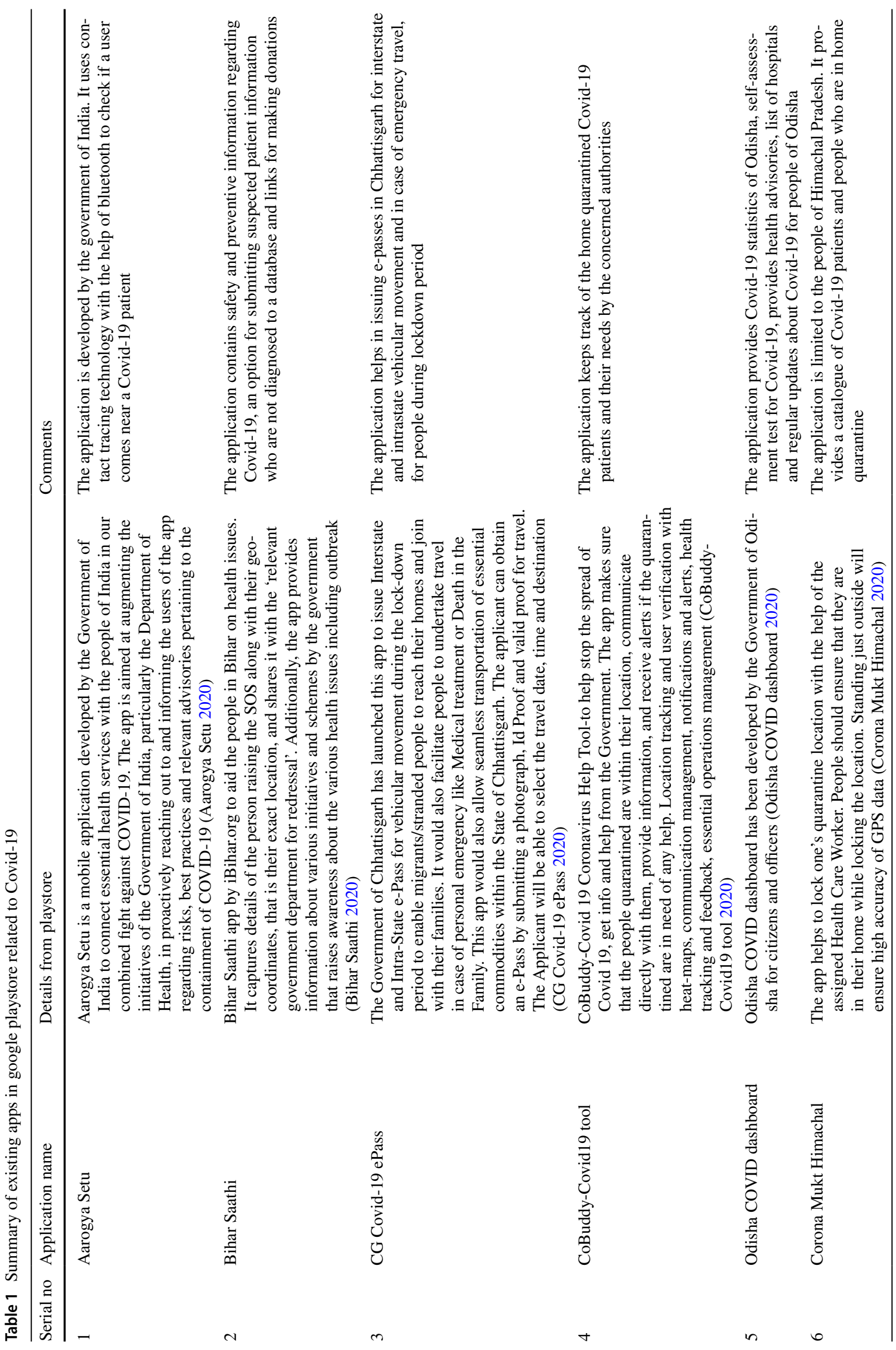




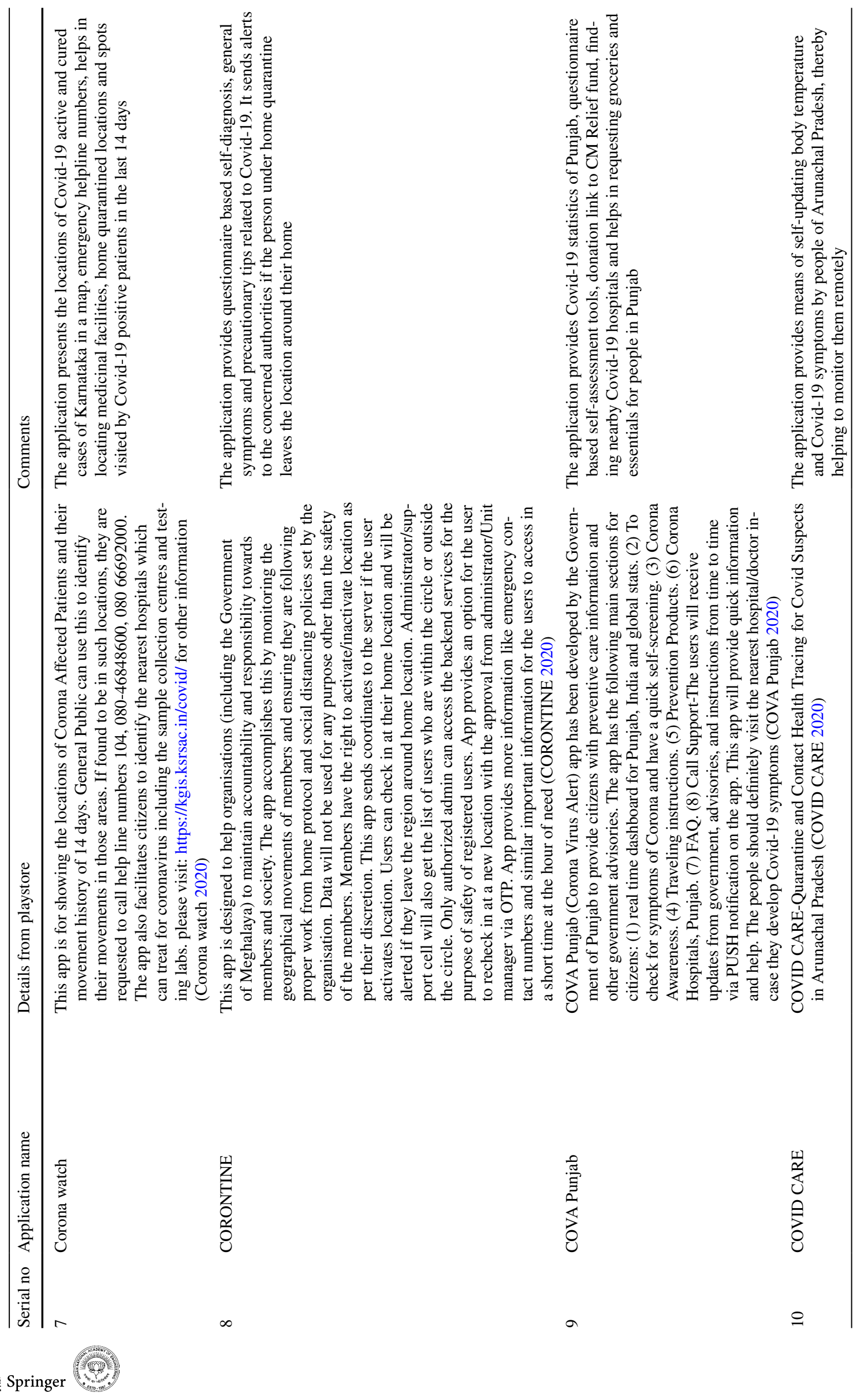




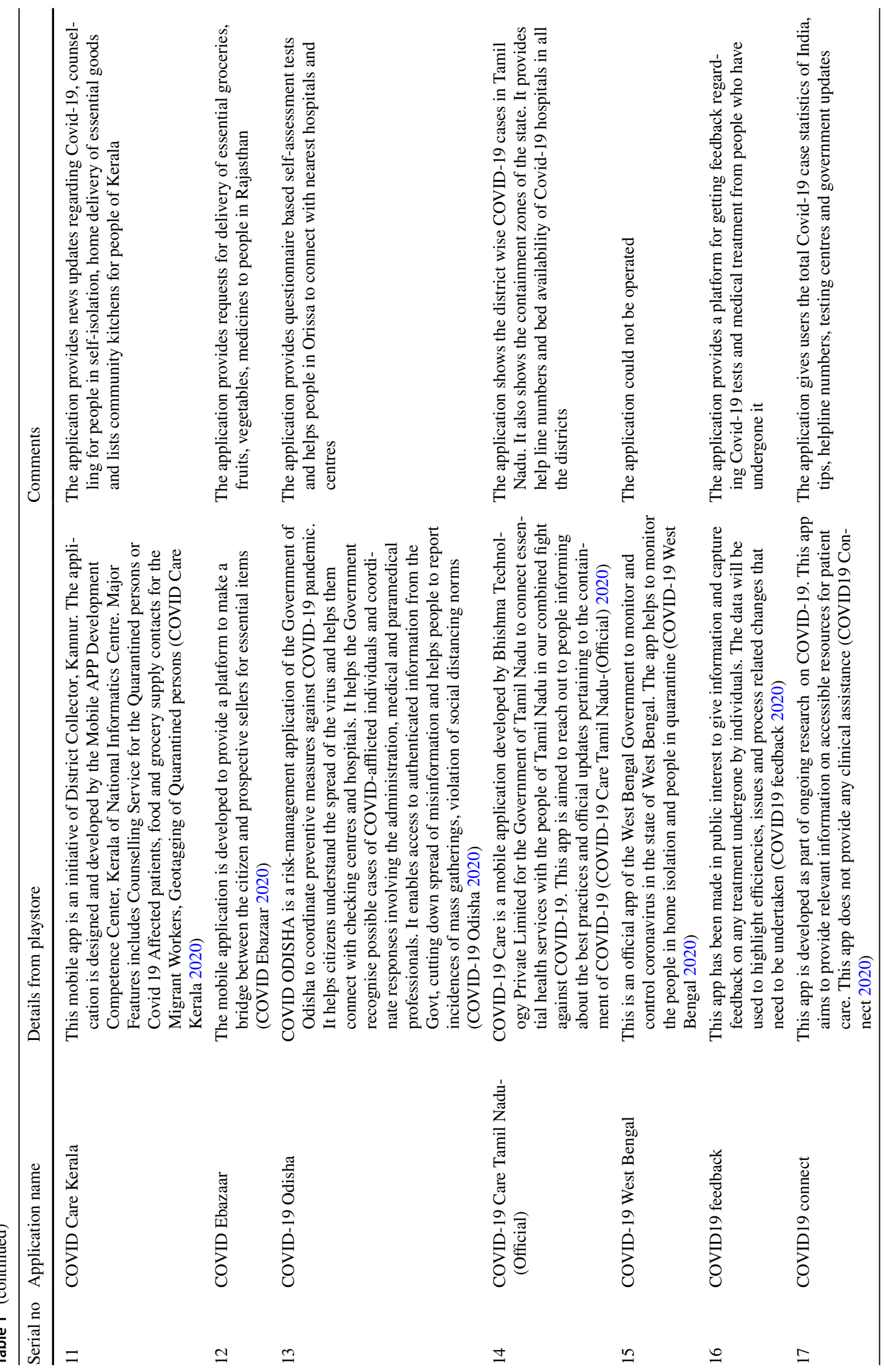




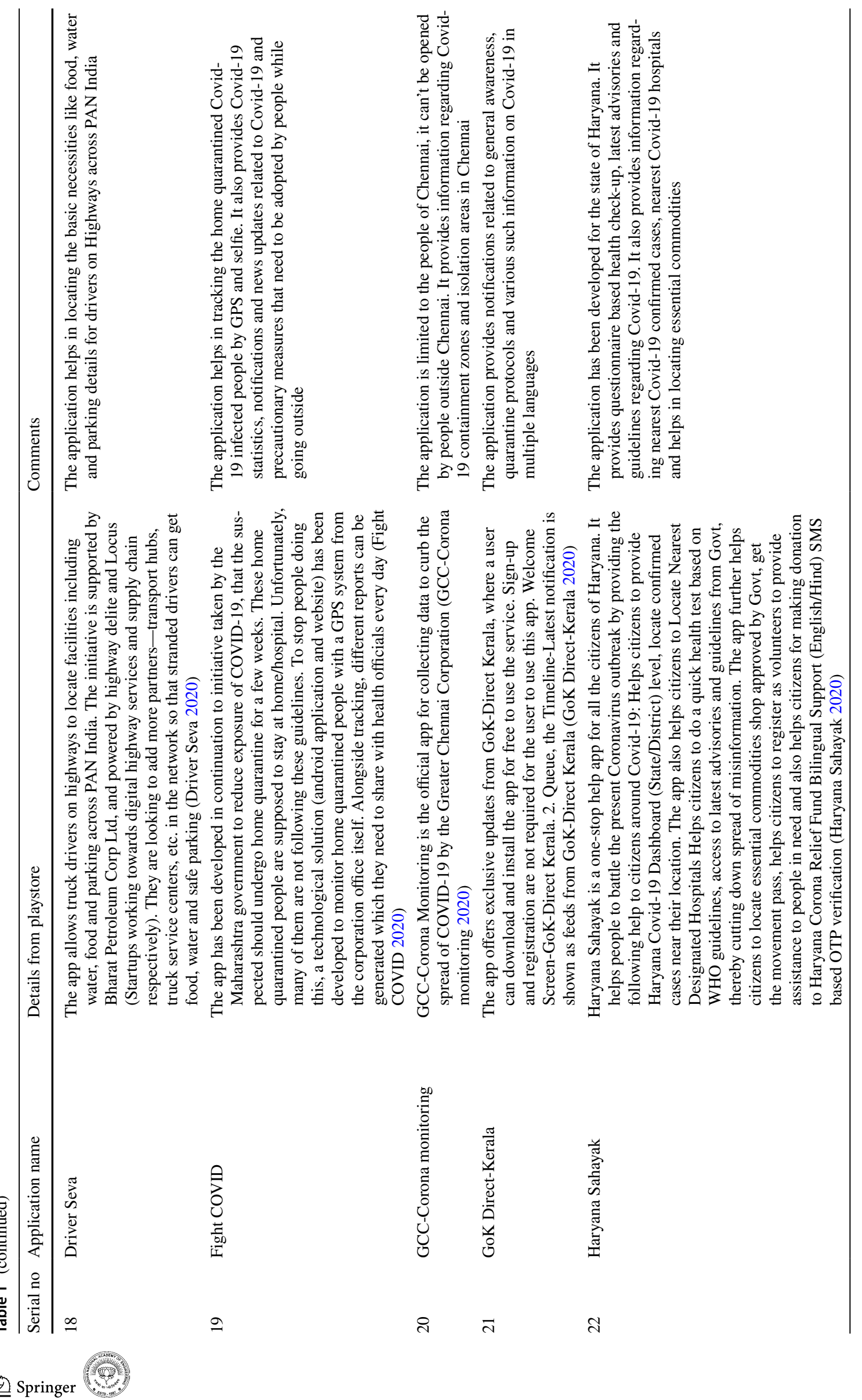




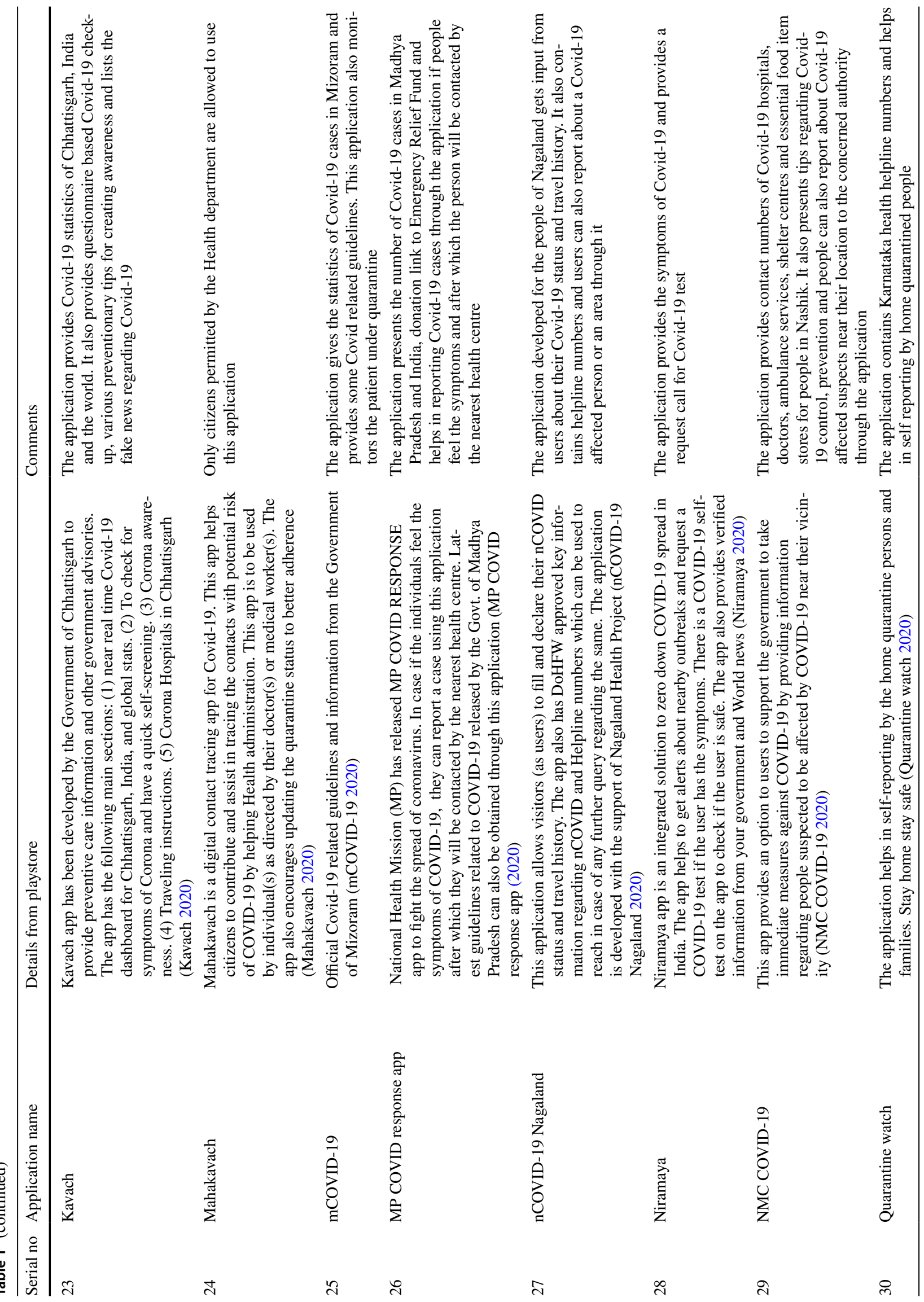




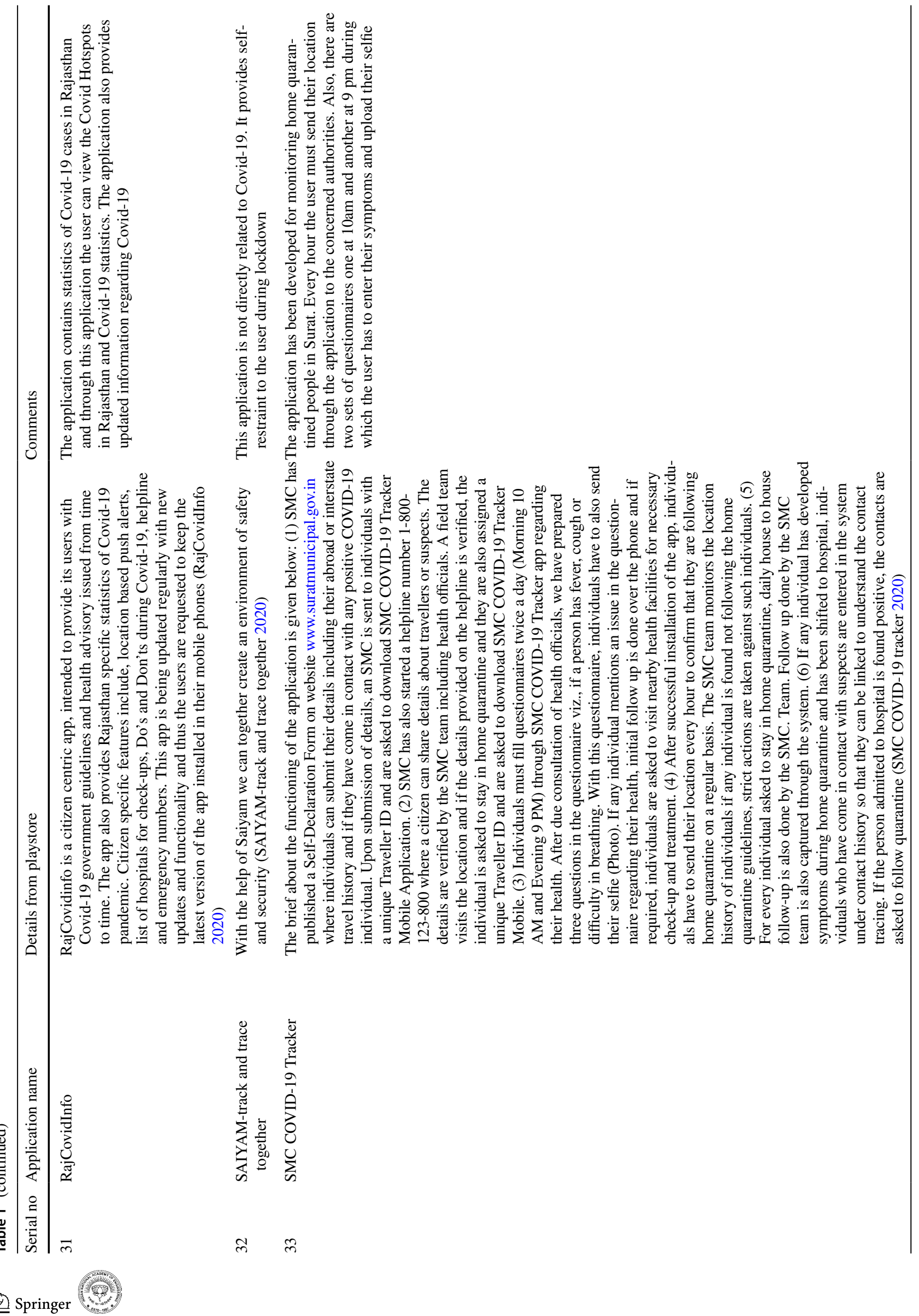




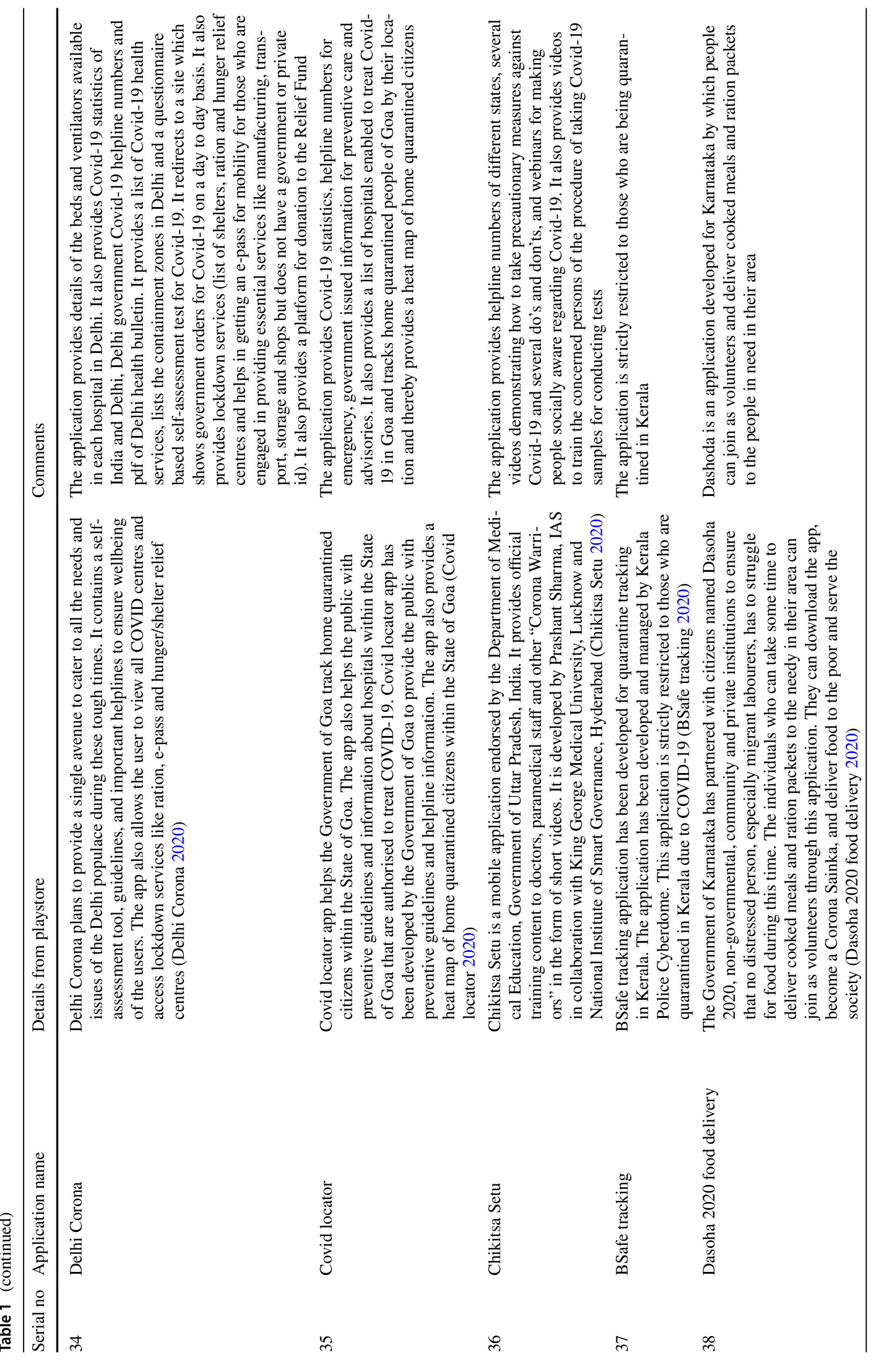



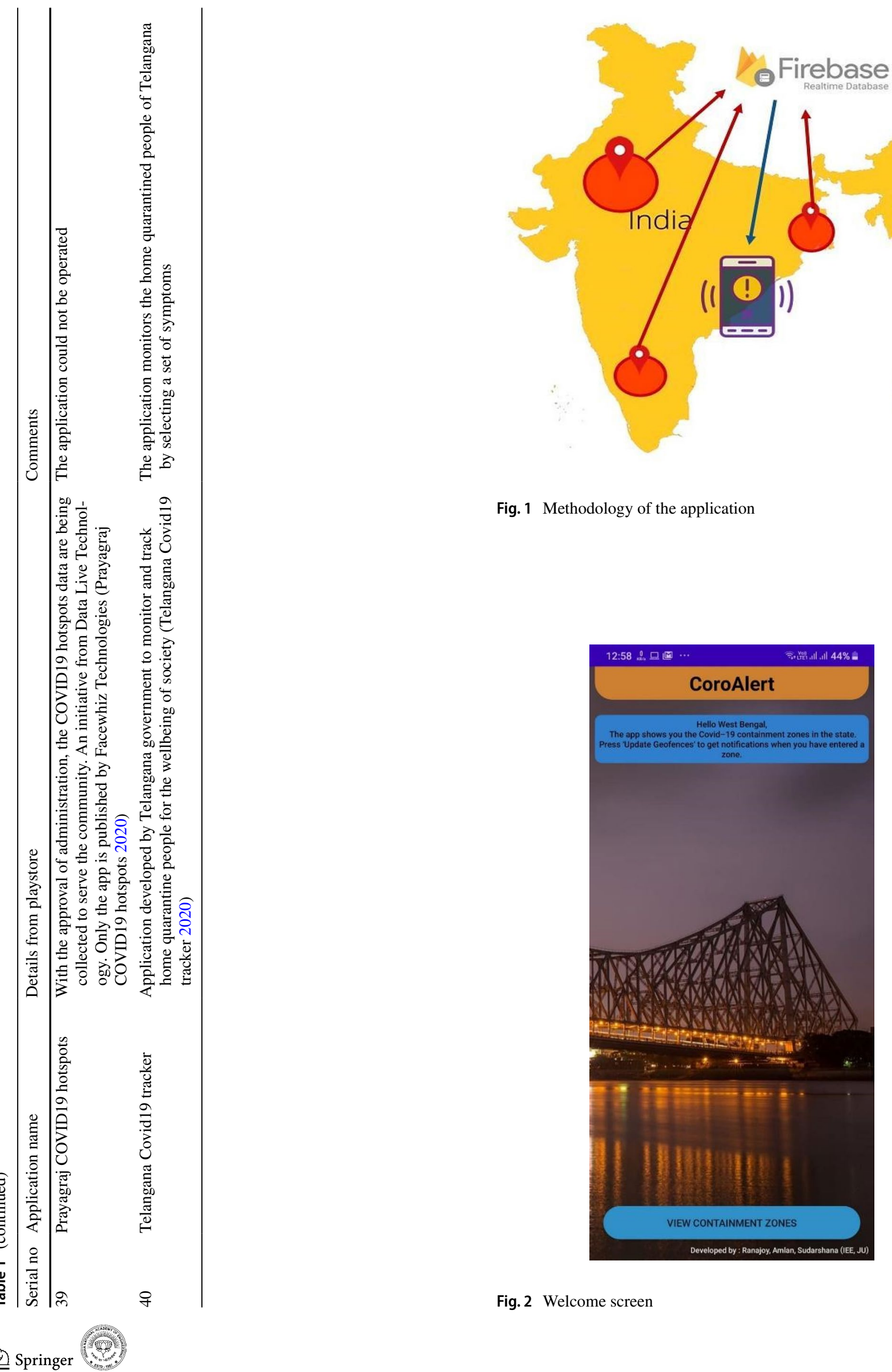

Fig. 1 Methodology of the application

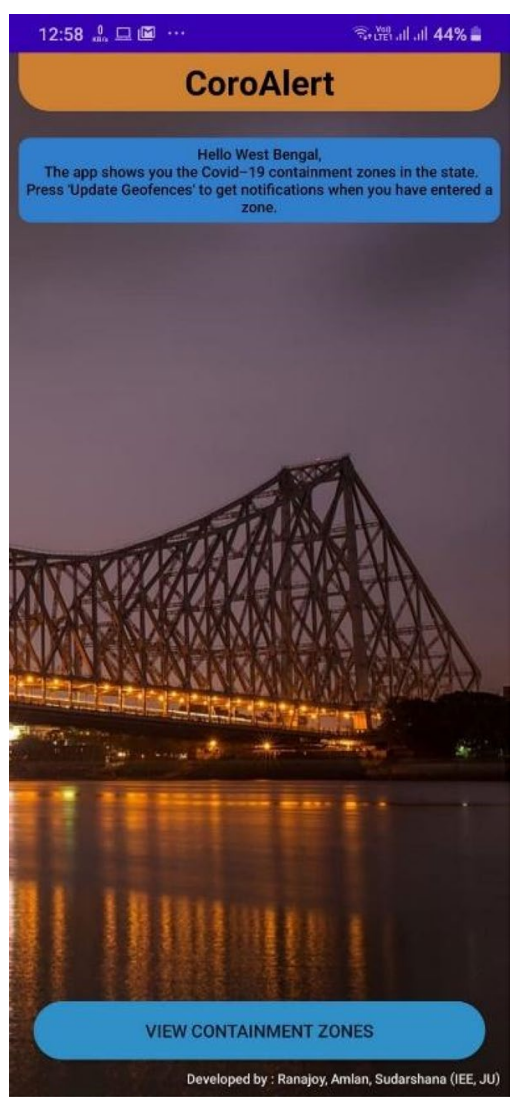

Fig. 2 Welcome screen 


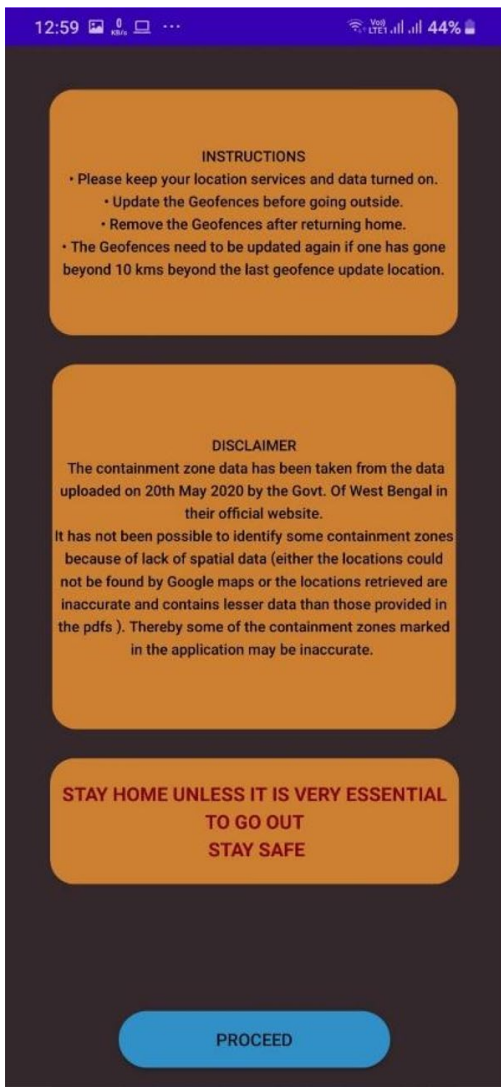

Fig. 3 Disclaimer screen

\section{Geofencing}

Geofencing API from Android is used to create virtual boundaries or fences around geographical locations (Create and monitor geofences 2020). The developers can add geofences at different locations by providing the latitudes and longitudes along with radius to define the virtual boundary at that location. Geofencing technology senses the user's current location and checks whether the location is inside any of the geofences created. A broadcast receiver receives intent contained in a pendingintent (an android API) sent by the location services when the user has entered, dwelt, or exited a geofence as shown in Fig. 6 and can initiate a background work or send a notification. The geofence transitions events include enter, exit, and dwell and multiple transition events can be set for the geofences. In this application, the dwell transition is set for the containment zones with a loitering delay of $5 \mathrm{sec}-$ onds and an expiration duration set to never expire. The broadcast receiver is set to initiate a notification by the notification manager upon receiving an intent. Once the

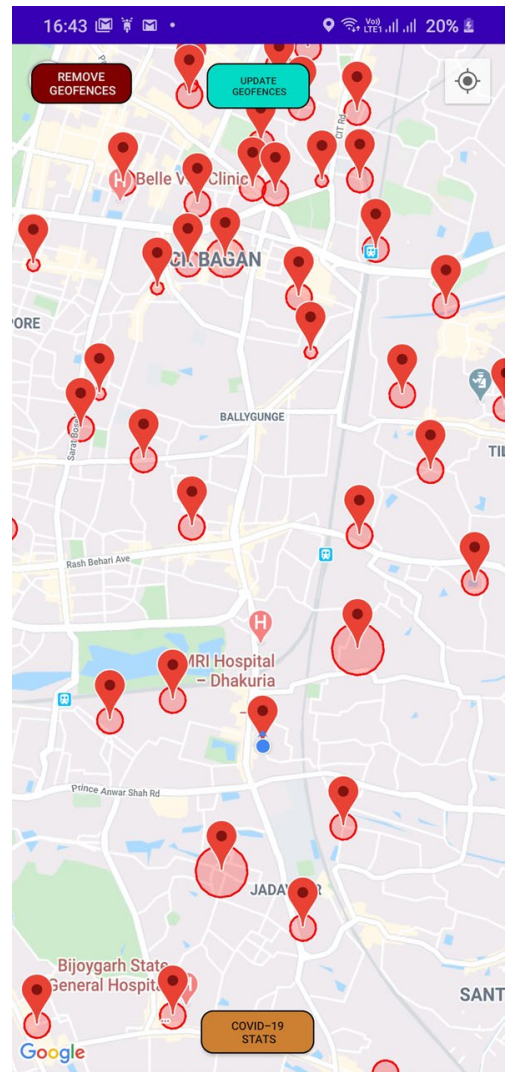

Fig. 4 Google map showing containment zones

geofences are set, the user would receive notification on entering and dwelling inside a containment zone.

\section{RESTful API}

Representational State Transfer (REST) API or RESTful web services are architectural styles for communications often used in web services development (RESTful API 2020). These APIs use less bandwidth than the Simple Object Access Protocol (SOAP) and hence they are useful for cloud applications. The RESTful API uses the HTTP methodologies which are defined by the RFC 2616 protocol. The information stored in a RESTful API are resources which can be read, updated, or deleted using resource methods like GET, POST or DELETE. The resources are accessed using Uniform Resource Identifiers (URIs). In this application, we have used a RESTful API from COVID19 India API (COVID19 India API 2020) and the resource that we have used is the Country and State wise data. We have used the GET method to receive the data of West Bengal as a JSON object in the application and show it in a bottom sheet (Fig. 7). 


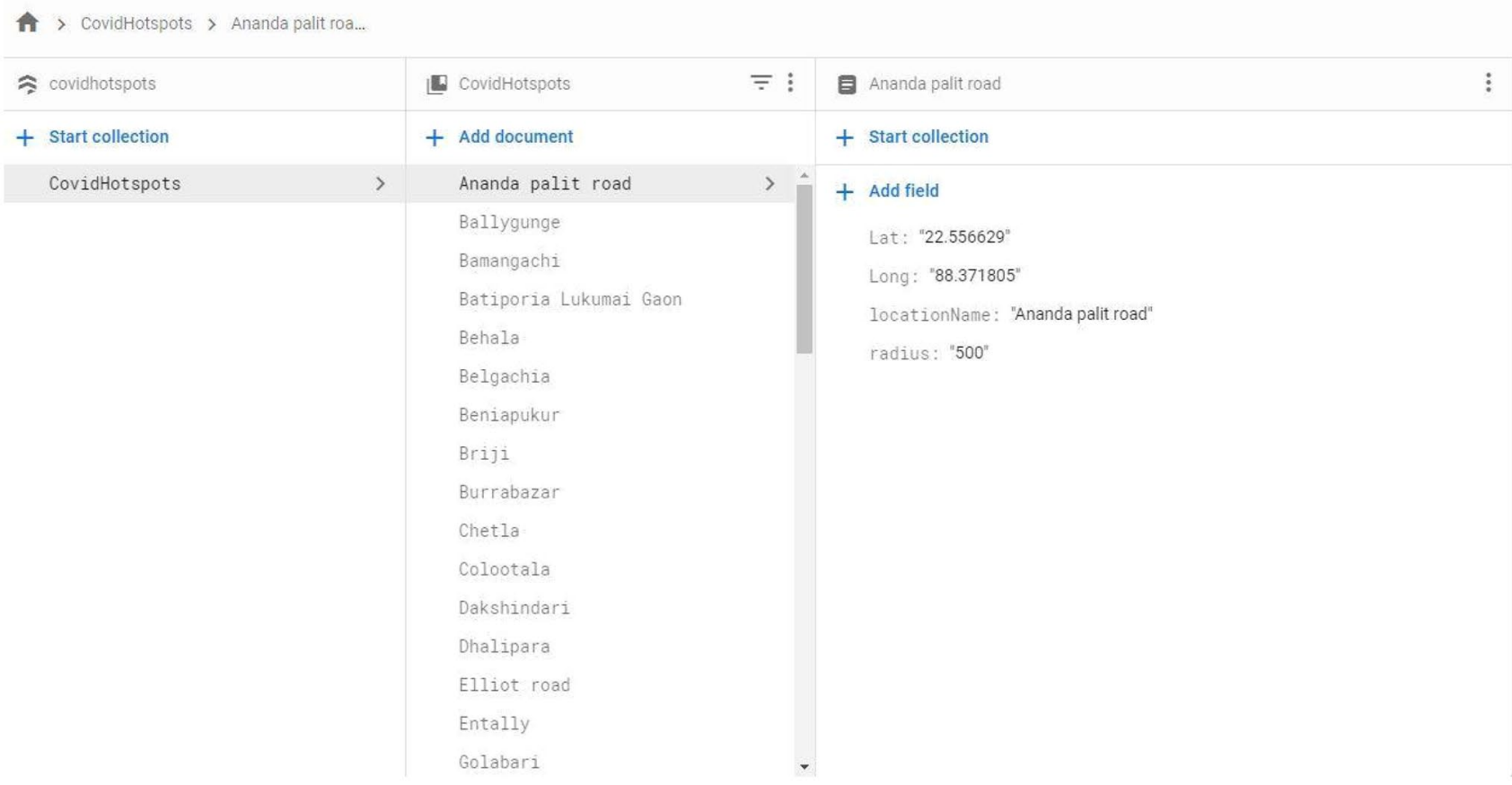

Fig. 5 Cloud Firestore database with location data of containment zones

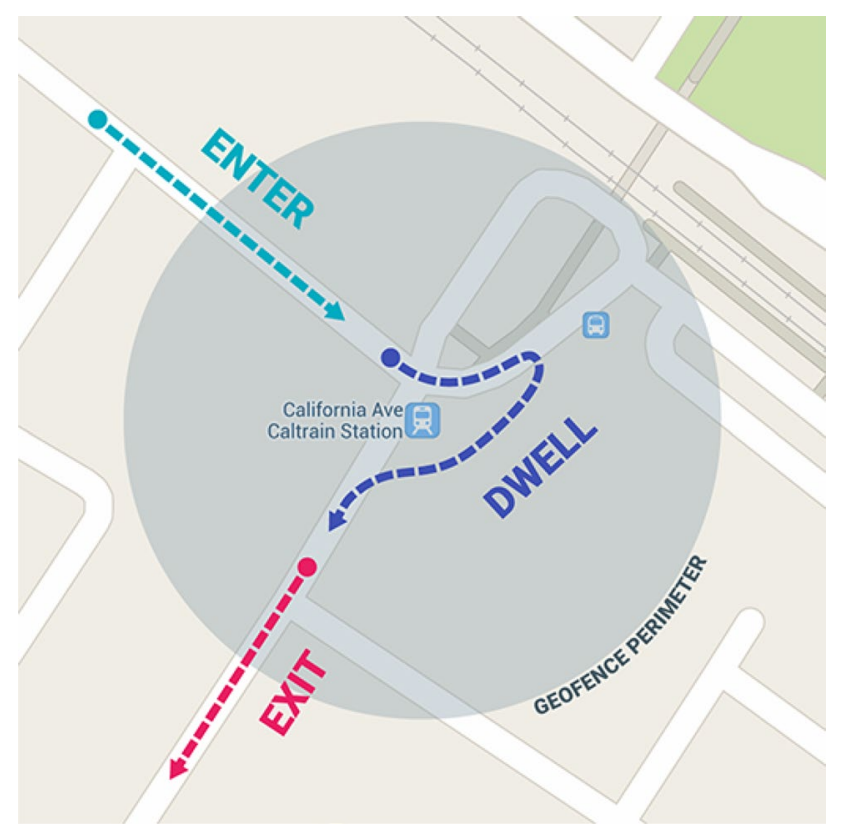

Fig. 6 Geofence trigger events

\section{Working of the Application}

The application gets data from the Cloud Firestore database. A collection is created in Cloud Firestore with containment zones as documents. Each document has four fields: latitude, longitude, location name and radius. Accordingly, a Java

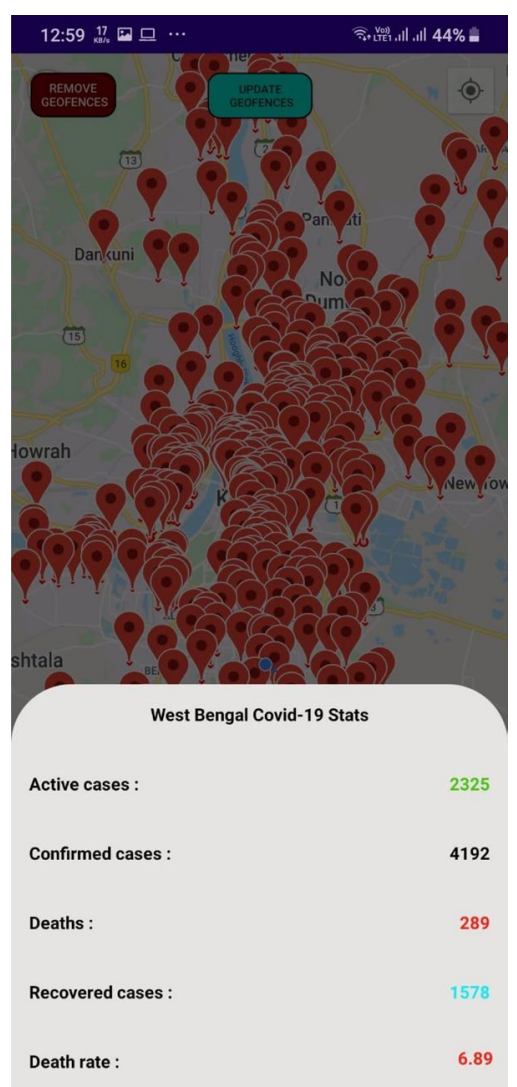

Fig. 7 Bottom sheet showing Covid-19 statistics of West Bengal 


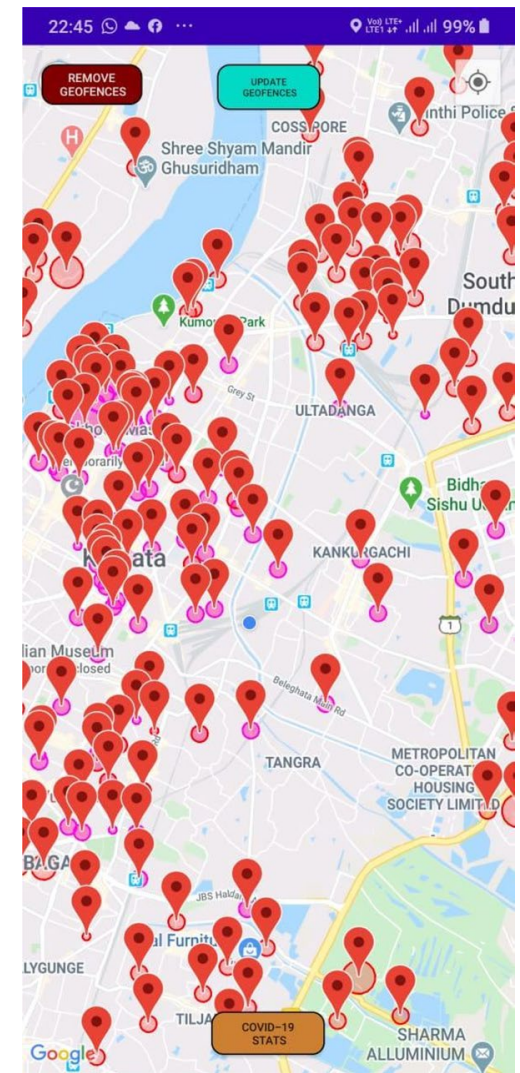

Fig. 8 Selection of closest containment zones for geofencing

object is created which can get the data from the document. In the map's activity, the firebase Firestore instance and collection references are created to which a snapshot listener is attached. The snapshot listener retrieves the document snapshots which are then converted into the Java object mentioned earlier. With the help of getters each data from the document is retrieved and are converted to string. Markers and circles are set using the location coordinates and radius and tags are given by the location names. The google map gets populated with these markers surrounded by circles which represents the containment zones. A JSON request is made with the get method to the REST API URL which returns the West Bengal Covid-19 case data as a response. The response is converted to a JSON object and the information is extracted. The google map shows all the containment zones in West Bengal along with the location of the user using "set my location" enabled (Google map API). The Geofencing API can create up to 100 geofences per device and the number of containment zones are more than 1000 in West Bengal. The solution to this problem is to create

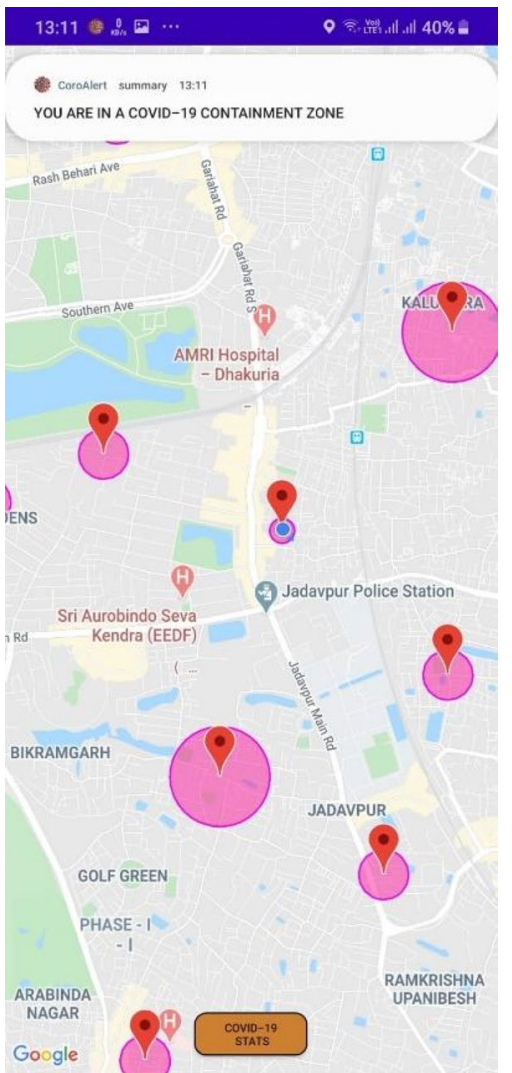

Fig. 9 Notifications from geofence broadcast

100 geofences on the 100 nearest containment zones. Once the map is loaded and populated with containment zones and user's location, the user can press a button to add the geofences on the closest 100 containment zones. The snapshot listener returns the documents containing the locations of the containment zones from Firestore which are not sorted according to the distance between the user and containment zone. The distance between the user and each containment zone is measured using distance between method of the location manager (Android developer-Locations 2020). This distance is then used as a key and is stored along with the document in a Tree map. Likewise, all the containment zones with their distance from the user get stored in the tree map and get sorted according to the distance or key. First 100 entries from the tree map are retrieved and geofences are created on these 100 containment zones as shown in Fig. 8 . Once the geofences are set, the user can get notification on entering the containment zones as shown in Fig. 9. The geofences get triggered and notifications are sent even when the app is not running in background as shown in Fig. 10. 
Fig. 10 Notifications without app running

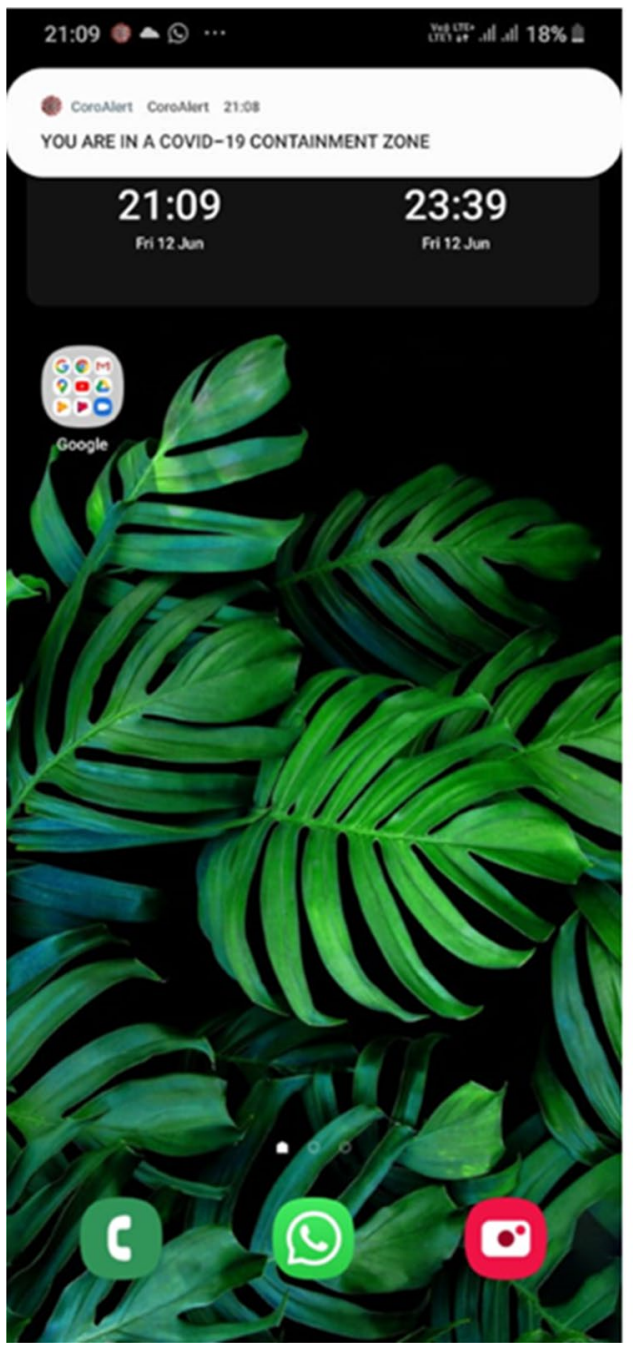

It is advised to remove the geofences when the user is not moving outside his or her residence to conserve power. The workflow of the app is shown in Fig. 11.

The application further extracts the IMEI number of the trespasser and uploads it to the online database. The application prompts background location services permission and if granted the geofences get triggered even if the application is not open in foreground.

\section{Results and Discussions}

Tests have been carried out in various containment zones across West Bengal for the validation of the Android application. The identified containment zones chosen for the testing of the application were visited one by one. Table 2 shows various containment zones identified for conducting the test, the date, time of entry, time of receiving the notification alerts upon entering. From Table 2, it is highlighted that the application sends notification alerts within 5-8 seconds on entering.

\section{Memory Utilization}

The memory profiler has been used to analyse the memory allocation of the application. It has been checked several times that the MapActivity is allocating around $150 \mathrm{MB}$ in which graphics takes around $40 \mathrm{MB}$ and Java $35 \mathrm{MB}$ and the graph is flat as shown in Fig. 12. Heap dump is recorded several times and no memory leaks have been found.

\section{Network Traffic}

The Network traffic is analysed using Network profiler in Android profiler and programmatically with Traffic stats method. The application uses the network to load the Google 


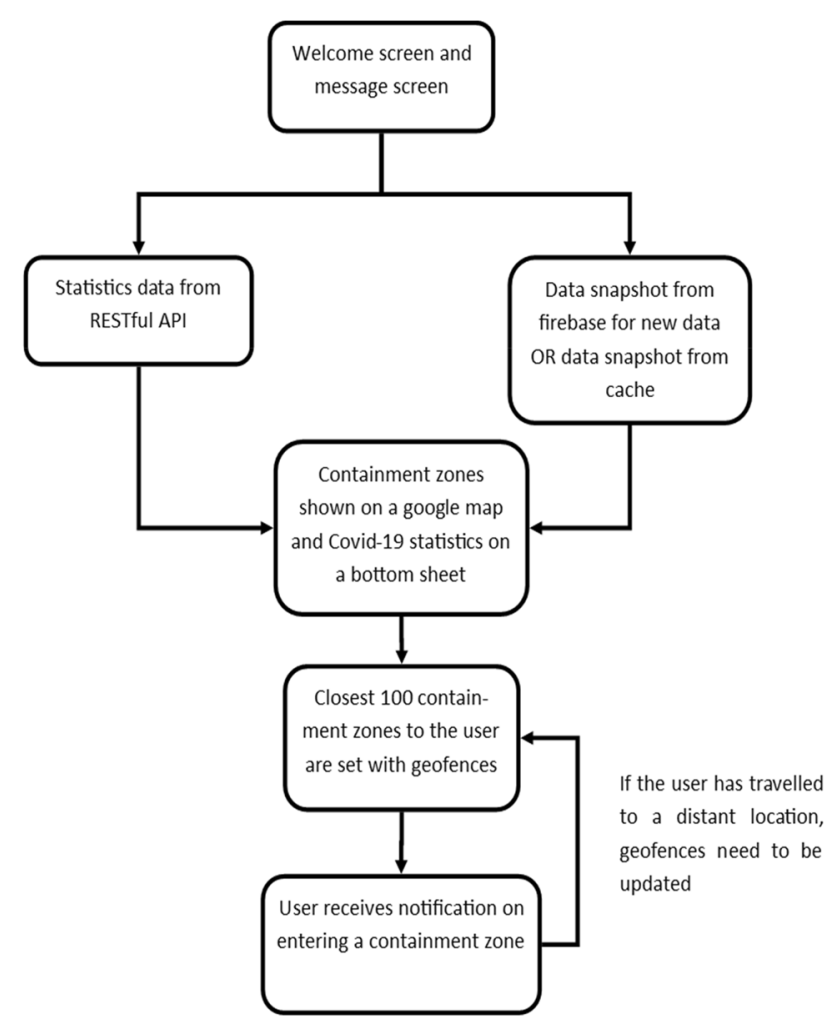

Fig. 11 Workflow of the application

maps, to retrieve containment zone data from the Firebase Cloud Firestore and to get Covid-19 statistics from a RESTful Api. Network usage spikes have been detected in Network profiler for MapsActivity and connections are attached from here. The receiving network bandwidth has been checked multiple times and is found to be around $1 \mathrm{MB} / \mathrm{s}$. The getUidRxBytes and getUidTxBytes methods of TrafficStats have been also used to monitor how much data the application is sending and receiving. First the application was installed on two devices and then 2 types of readings were taken. In the first reading shown in Table 3, the application was initiated several times without destroying the application process in the background. In the second reading, the application process was destroyed before taking each reading (Table 4 ).

It has been observed that the application receives around $23 \mathrm{~KB}$ of data during the start of the MapsActivity when the application is initiated and receives around $10 \mathrm{~KB}$ when it has been running in the background. This data includes both Firestore data and Google maps data.

\section{Conclusion and Future Scopes}

The application provides an efficient way of showing the identified Covid-19 containment zones to the users in a Google map. With the alarming increase of Covid-19 affected cases throughout the world, this developed application can be employed as a tool for creating further social awareness among the people. This application further tracks the user's location and checks whether it is present in the list of identified containment zones. It sends separate notification alerts to the user on entering. The developed android application further extracts the IMEI Number of the trespasser in the containment zones which can be useful to the local police to track and identify people who are frequently trespassing the containment zones. Thereby this application identifies the containment zones and highlights the need for taking further precautionary measures for combating Covid19. The application has been tested in various locations and has been found to yield accurate results.

The application can be further used for many purposes like maritime and forest safety to prevent users from entering restricted areas.

Table 2 Containment zones with the time of entry, the time of receiving notification alerts in the android application and the extracted IMEI number of the trespasser in the corresponding containment zones which is uploaded to the online database

\begin{tabular}{llllll}
\hline Serial number & $\begin{array}{l}\text { Name of the contain- } \\
\text { ment zone }\end{array}$ & $\begin{array}{l}\text { Date of entry in the } \\
\text { containment zone }\end{array}$ & $\begin{array}{l}\text { Time of entry in the } \\
\text { containment zone }\end{array}$ & Notification time & IMEI number \\
\hline 1 & Bamangachi & 21.04 .2020 & $09: 30: 16$ & $09: 30: 21$ & 356129106619221 \\
2 & Belgachia & 20.04 .2020 & $09: 45: 50$ & $09: 45: 55$ & 356129106619221 \\
3 & Garden reach & 22.04 .2020 & $10: 55: 10$ & $10: 55: 18$ & 868134038083620 \\
4 & Garia & 22.04 .2020 & $11: 52: 19$ & $11: 20: 59$ & 356129106619221 \\
5 & Golabari & 21.04 .2020 & $11: 20: 50$ & $10: 42: 25$ & 356129106619221 \\
6 & Kaikhali & 20.04 .2020 & $10: 42: 12$ & $09: 46: 08$ & 868134038083612 \\
7 & Mudiali & 23.04 .2020 & $09: 46: 00$ & $10: 58: 28$ & 356129106619221 \\
8 & Nayabad & 23.04 .2020 & $10: 58: 21$ & $11: 35: 17$ & 356129106619221 \\
9 & Park circus & 20.04 .2020 & $11: 35: 12$ & $09: 35: 30$ & 868134038083612 \\
10 & Tikiapara & 22.04 .2020 & $09: 35: 23$ & & 868134038083612 \\
\hline
\end{tabular}




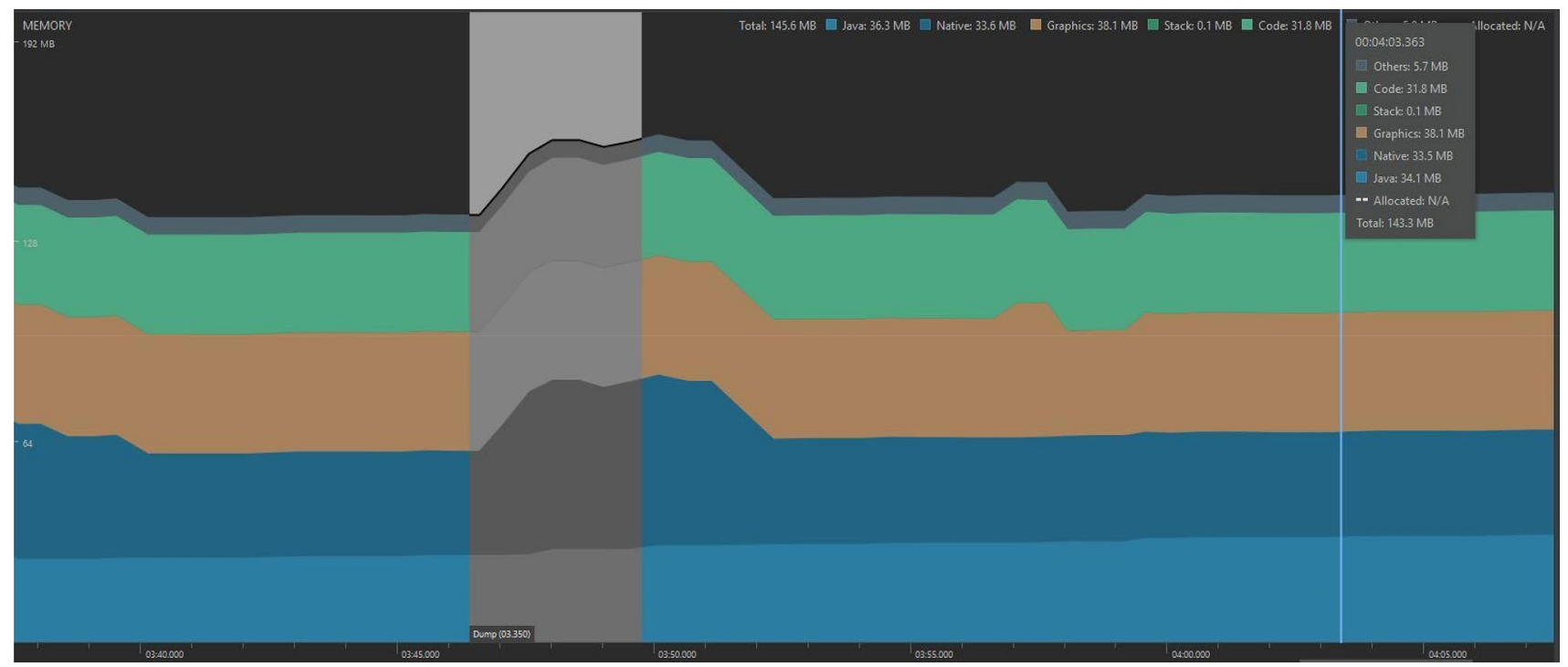

Fig. 12 Memory utilization

Table 3 Bytes received application initiating

\begin{tabular}{lcc}
\hline Test no & Bytes received by device 1 & $\begin{array}{c}\text { Bytes received } \\
\text { by Device 2 }\end{array}$ \\
\hline 1 & 12,273 & 11,706 \\
2 & 9413 & 10,476 \\
3 & 9644 & 9908 \\
4 & 7199 & 10,020 \\
5 & 12,803 & 9909 \\
6 & 10,693 & 10,019 \\
7 & 10,112 & 9174 \\
8 & 9399 & 9920 \\
9 & 9497 & 9899 \\
10 & 9499 & 9571 \\
\hline
\end{tabular}

Table 4 Bytes received when app starts from background

\begin{tabular}{lll}
\hline Test no & Bytes received by device 1 & $\begin{array}{l}\text { Bytes } \\
\text { received by } \\
\text { device } 2\end{array}$ \\
\hline 1 & 22,644 & 25,787 \\
2 & 22,634 & 22,130 \\
3 & 22,184 & 21,321 \\
4 & 27,604 & 21,363 \\
5 & 22,317 & 23,397 \\
6 & 23,234 & 23,779 \\
7 & 22,307 & 24,739 \\
8 & 22,030 & 23,110 \\
9 & 24,993 & 22,529 \\
10 & 22,342 & 22,915 \\
\hline
\end{tabular}

Author Contributions All the authors have contributed in their respective parts.

Funding Not applicable.

Availability of Data and Material Data and material available.

\section{Compliance with ethical standards}

Conflict of interest There are no conflicts of interest.

Code availability The Java code of the app is available.

\section{References}

Aarogya Setu (2020) https://play.google.com/store/apps/details?id=nic. goi.aarogyasetu\&hl=en

Android developer-Locations (2020) https://developer.android.com/ reference/android/location/Location\#distanceBetween(doubl e, \%2520double, $\% 2520$ double, \%2520double,\%2520float[])

BSafe tracking (2020) https://play.google.com/store/apps/detai ls? id=com.cyberdome.bsafe \&hl=en

Bahl P, Doolan C, de Silva C, Chughtai AA, Bourouiba L, MacIntyre CR (2020) Airborne or droplet precautions for health workers treating Coronavirus disease 2019? J Infect Dis. https://doi. org/10.1093/infdis/jiaa189

Bihar Saathi (2020) https://play.google.com/store/apps/details?id=com. ibihar.saathi\&hl=en

CG Covid-19 ePass (2020) https://play.google.com/store/apps/detai ls?id=com.allsoft.corona\&hl=en

Chikitsa Setu (2020) https://play.google.com/store/apps/detai ls? $\mathrm{id}=$ com. abhitech.chikitsasetu\&hl=en

Cloud Firestore Data model (2020) https://firebase.google.com/docs/ firestore/data-model

Cloud Firestore (2020) https://firebase.google.com/docs/Firestore 
Cloud Firestore SDKs and client libraries (2020) https://firebase.googl e.com/docs/Firestore/client/libraries

CoBuddy-Covid19 tool (2020) https://play.google.com/store/apps/detai ls?id=www.facetagr.com.cobuddy\&hl=en

Corona Mukt Himachal (2020) https://play.google.com/store/apps/detai ls? id=com.developmentlogics.patientgeotracker\&hl=en

Corona watch (2020) https://play.google.com/store/apps/detai $1 \mathrm{~s}$ ?id=com.ksrsac.drawshapefile \&hl=en

Covid locator (2020) https://play.google.com/store/apps/detai ls?id=com.intutrack.covidtrack\&hl=en

CORONTINE (2020) https://play.google.com/store/apps/detai ls? id=com.itakash.iitbc \&hl=en

COVA Punjab (2020) https://play.google.com/store/apps/detai ls?id=in.gov.punjab.cova\&hl=en

COVID-19 Odisha (2020) https://play.google.com/store/apps/detai ls?id=odisha.gov.covid $19 \&$ hl=en

COVID-19 Care Tami Nadu-(Official) (2020) https://play.googl e.com/store/apps/details?id=com.rvmatrix.corona\&hl=en

COVID-19 West Bengal (2020) https://play.google.com/store/apps/ details?id=com.pixxonai.covid $19 \mathrm{wb} \& \mathrm{hl}=\mathrm{en}$

COVID19 feedback (2020) https://play.google.com/store/apps/detai ls? id=com.NIC.covid $19 \& \mathrm{hl}=\mathrm{en}$

COVID19 Connect (2020) https://play.google.com/store/apps/detai ls? id=com.igib.COVID19Connect\&hl=en

COVID19 India API (2020) https://covid-19india-api.herokuapp. $\mathrm{com} /$

COVID CARE (2020) https://play.google.com/store/apps/detai ls?id=app.igotit.covidcare \&hl=en

COVID Care Kerala (2020) https://play.google.com/store/apps/detai ls?id=org.nic.covidcarekannur\&hl=en

COVID Ebazaar (2020) https://play.google.com/store/apps/detai ls?id=gov.ebazaar.covid\&hl=en

Create and monitor geofences (2020) https://developer.android.com/ training/location/geofencing.html\#HandleGeofenceTransitions

Dasoha 2020 food delivery (2020) https://play.google.com/store/ apps/details?id=in.swiggy.covidvolunteer\&hl=en

Delhi Corona (2020) https://play.google.com/store/apps/detai ls? $\mathrm{id}=\mathrm{com}$. delhi.covidcare $\& \mathrm{hl}=\mathrm{en}$

Driver Seva (2020) https://play.google.com/store/apps/details?id=com. hd. seva\&hl=en

Fight COVID (2020) https://play.google.com/store/apps/detai $\mathrm{ls} ? \mathrm{id}=$ com.pratikthorat.coronatracker\&hl=en
GCC-Corona monitoring (2020) https://play.google.com/store/apps/ details?id=com.gcc.smartcity \&hl=en

GoK Direct-Kerala (2020) https://play.google.com/store/apps/detai ls?id=com.qkopy.prdkerala\&hl=en

Haryana Sahayak (2020) https://play.google.com/store/apps/detai ls?id=hr.gov.covid19.sahayak\&hl=en

Kavach (2020) https://play.google.com/store/apps/details?id=in.gov. chhattisgarh.cova\&hl=en

Mahakavach (2020) https://play.google.com/store/apps/detai ls?id=com.mahakavach\&hl=en

mCOVID-19 (2020) https://play.google.com/store/apps/details?id=gov. mizoram.mcovid19\&hl=en

MP COVID response app (2020) https://play.google.com/store/apps/ details?id=com.covidmp.coronago\&hl=en

nCOVID-19 Nagaland (2020) https://play.google.com/store/apps/detai ls? id=com.tattvafoundation.ncovid \&hl=en

Niramaya (2020) https://play.google.com/store/apps/details?id=com. niramaya.people\&hl=en

NMC COVID-19 (2020) https://play.google.com/store/apps/detai ls?id=com.kodwell.nmccovid_19\&hl=en

Odisha COVID dashboard (2020) https://play.google.com/store/apps/ details?id=com.ocac.covidodisha\&hl=en

Prayagraj COVID19 hotspots (2020) https://play.google.com/store/ apps/details?id=com.prayagraj.covid19.hotspots\&hl=en

Quarantine watch (2020) https://play.google.com/store/apps/detai ls?id=com.bmc.qrtnwatch\&hl=en

RajCovidInfo (2020) https://play.google.com/store/apps/detai ls? id=com.gismcg.covid19_rajasthan\&hl=en

RESTful API (2020) https://searchapparchitecture.techtarget.com/defin ition/RESTful-API\#: :text=A\%2520RESTful\%2520API\%2520i s\%2520an,to\%2520communicate $\% 2520$ with $\% 2520$ each $\% 2520$ o ther

SAIYAM-track and trace together (2020) https://play.google.com/store /apps/details?id=com.flowace. saiyam\&hl=en

SMC COVID-19 tracker (2020) https://play.google.com/store/apps/ details?id=in.smc.covidout\&hl=en

Telangana Covid19 tracker (2020) https://play.google.com/store/apps/ details?id=com.corona.tscovid_19\&hl=en

Publisher's Note Springer Nature remains neutral with regard to jurisdictional claims in published maps and institutional affiliations. 